sche Parlament in der Plenarsitzung am 03.04.2014 der von der EUKommission vorgeschlagenen eIDAS-Verordnung zugestimmt. Damit sind umfangreiche Änderungen von Inhalt und Wortlaut verbunden, die nun von der Kommission in den finalen Verordnungsentwurf übernommen werden, der dann der abschließenden Trilog Verhandlung zugrunde liegen wird.

Das Abstimmungsergebnis im Parlament:

Zustimmung 534

Ablehnung 73

Enthaltungen 7

\section{Mobiles Geschütztes Fernmeldeaufklärungssystem in Daun: DS eingehalten}

Die Bundesbeauftragte für den Datenschutz und die Informationsfreiheit Andrea Voßhoff konnte bei ihrem Besuch keine Datenschutzverletzung durch den Testeinsatz des Mobilen Geschützten Fernmeldeaufklärungssystems (MoGeFA) am Bundeswehrstandort in Daun feststellen.

Am 26. März 2014 haben Mitarbeiter der Bundesbeauftragten das MoGeFA am Bundeswehrstandort Daun in der Eifel kontrolliert. Anlass waren Pressemeldungen und die Anfragen eines Bundestagsabgeordneten zum Datenschutz beim MoGeFA in der Fragestunde des Deutschen Bundestages am 19. Februar 2014. Unklar war in diesem Zusammenhang insbesondere, ob zivile Kommunikation im Umfeld durch dieses System mitgehört werden kann.

Bei dieser Kontrolle wurde festgestellt, dass das Testmodell des Mobilen Geschützten Fernmeldaufklärungssystems keine zivile Kommunikation erfasst. Die in Daun vorgesehenen Tests betreffen ausschließlich militärische Frequenzen. Ferner sind technische und organisatorische Vorkehrungen dafür getroffen worden, damit keine sensible zivile Kommunikation empfangen werden kann. Die Bundeswehr würde nur dann zivilen Funkverkehr erfassen, wenn von Bürgern missbräuchlicherweise Funkfrequenzen der Bundeswehr genutzt würden. Allerdings hat die Bundeswehr auch für diesen Fall Vorkehrungen getroffen, solche Daten unverzüglich zu löschen.

Andrea Voßhoff: „Ich sehe derzeit keine datenschutzrechtliche Gefährdung der Dauner Bevölkerung durch den Testeinsatz. Ich beabsichtige, die verschiedenen Entwicklungsphasen des MoGeFA weiterhin zu begleiten."

\section{FireEye: Neuer Mandiant Threat Report}

FireEye, Inc., Experte bei der Abwehr von bisher unbekannten Cyberangriffen, veröffentlichte am 11.04.2014 den nunmehr fünften jährlichen Mandiant M-Trends Report. Der Report „Beyond the Breach" basiert auf intensiven Forschungsaktivitäten und Analysen von Mandiant aus dem Jahr 2013. Er zeigt die Taktiken und Methoden der Angreifer auf, mit denen sich diese einen Zugriff auf die sensiblen Daten von Unternehmen verschaffen wollen. Er verweist darüber hinaus auf kommende, global auftretende neue Gefahren, die vermutlichen Motive der Cyberkriminellen sowie ihre bevorzugten Opfer.
Auf Basis der von Mandiant im Jahr 2013 untersuchten Vorfälle bietet „Beyond the Breach“ einige wichtige Erkenntnisse:

Die Zeit bis zur Entdeckung von Angriffen schrumpft weiter Die durchschnittliche Zeit, in der sich Angreifer ungehindert in Firmennetzwerken bewegen konnten, sank von 243 Tagen im Jahr 2012 auf 229 Tage im Jahr 2013. Der Rückgang scheint angesichts des entsprechenden Verhältnisses von 2011 auf 2012 (von 416 auf 243 Tage) vergleichsweise geringer. In jedem Fall aber können sich Unbefugte unter Umständen jahrelang unentdeckt in Unternehmensnetzwerken herumtreiben - in einem konkreten Fall sogar sechs Jahre und drei Monate.

Unternehmen müssen generell ihre Fähigkeiten bei der Entdeckung von Angriffen verbessern

Im Jahr 2012 hatten noch 37 Prozent der Unternehmen eigenständig Attacken entdeckt - im Jahr 2013 waren es nur noch 33 Prozent.

Phishing E-Mails tarnen sich als Nachricht aus der Unternehmens-IT

44 Prozent der im Report erfassten Phishing E-Mails gaben sich als Nachricht der jeweiligen IT-Abteilungen in den Unternehmen aus. Die überwiegende Mehrheit dieser E-Mails wurde an einem Dienstag, Mittwoch oder Donnerstag versendet.

Cyberangriffe im Zuge von politischen Konflikten betreffen zunehmend auch Unternehmen

Im letzten Jahr hat Mandiant eine erhöhte Zahl von Vorfällen festgestellt, wo Cyber-Angriffe im Rahmen zwischenstaatlicher Konflikte auch Unternehmen in den jeweiligen Ländern betrafen. Hierbei ist besonders die Syrian Electronic Army (SEA) zu erwähnen, die Webseiten und Social Media Accounts von privaten Organisationen manipulierte, um auf ihre politischen Ziele aufmerksam zu machen.

Mutmaßlich aus dem Iran stammende Angreifer spionieren bei Energieversorgern, Behörden und Regierungen

Intensive Untersuchungen im Energiesektor und bei Behörden haben erhöhte Cyber-Spionageaktivitäten gezeigt, deren Ursprung vermutlich im Iran liegt. Das legt den Schluss nahe, dass Angriffe zunehmend auch aus Überwachungsgründen geführt werden. Und obwohl die Angriffe aus dem Iran längst nicht auf dem neuesten technischen Stand sind, besteht kein Grund zu Beruhigung - die Angreifer werden ihre Methoden im Lauf der Zeit zweifelsohne verfeinern.

Eine Vollversion des 2014 Mandiant M-Trends-Reports ist erhältlich unter: www.mandiant.com/m-trends2014

\section{Projekt VeriMetrix: Kennzahlensystem zur Datenschutzbewertung von Cloud-Angeboten}

Wie sicher ist die Cloud? Viele Unternehmen und Behörden haben Bedenken, dass ihre Daten bei externen Cloud-Anbietern nicht ausreichend geschützt sind. Das Projekt VeriMetrix - über das am 09.04.2014 vom Fraunhofer SIT informiert wurde - macht deshalb die Sicherheit von Cloud-Daten messbar: Ziel von VeriMetrix ist eine Lösung, mit welcher potenzielle Nutzer Datenschutz und Da- 